\title{
Overexpression of the High Affinity Choline Transporter in Cortical Regions Affected by Alzheimer's Disease

\author{
Evidence from Rapid Autopsy Studies
}

\author{
T. A. Slotkin, ${ }^{\star}$ C. B. Nemeroff, ${ }^{\star}$ G. Bissette, ${ }^{\star}$ and F. J. Seidler* \\ *Departments of Pharmacology and Psychiatry, Duke University Medical Center, Durham, North Carolina 27710; and ${ }^{\ddagger}$ Department of \\ Psychiatry and Behavioral Sciences, Emory University School of Medicine, Atlanta, Georgia 30322
}

\begin{abstract}
Cholinergic deficits in Alzheimer's disease are typically assessed by choline acetyltransferase, the enzyme that synthesizes acetylcholine. However, the determining step in acetylcholine formation is choline uptake via a high affinity transporter in nerve terminal membranes. Evaluating uptake is difficult because regulatory changes in transporter function decay rapidly postmortem. To overcome this problem, brain regions from patients with or without Alzheimer's disease were frozen within $4 \mathrm{~h}$ of death and examined for both choline acetyltransferase activity and for binding of $\left[{ }^{3} \mathrm{H}\right]-$ hemicholinium-3 to the choline transporter. Consistent with the loss of cholinergic projections, cerebral cortical areas exhibited marked decreases in enzyme activity whereas the putamen, a region not involved in Alzheimer's disease, was unaffected. However, $\left[{ }^{3} \mathrm{H}\right]$ hemicholinium-3 binding was significantly enhanced in the cortical regions. In the frontal cortex, the increase in $\left[{ }^{3} \mathrm{H}\right]$ hemicholinium-3 binding far exceeded the loss of choline acetyltransferase, indicating transporter overexpression beyond that necessary to offset loss of synaptic terminals. These results suggest that, in Alzheimer's disease, the loss of cholinergic function is not dictated simply by destruction of nerve terminals, but rather involves additional alterations in choline utilization; interventions aimed at increasing the activity of cholinergic neurons may thus accelerate neurodegeneration. (J. Clin. Invest. 1994. 94:696-702.) Key words: acetylcholine, function in Alzheimer's disease - Alzheimer's disease • autopsy, rapid, in Alzheimer's disease • choline acetyltransferase, in Alzheimer's disease - choline transporter, in Alzheimer's disease
\end{abstract}

\section{Introduction}

Alzheimer's disease, morphologically characterized by the abnormal presence of senile plaques and neurofibrillary tangles, is distinguished neuroanatomically by the loss of cholinergic projections to the cerebral cortex, a deficit that has provided a focus for studies of potential therapies for cognitive defects (13 ). Unfortunately, the rapid postmortem degradation of acetyl-

Address correspondence to Dr. T. A. Slotkin, Box 3813/Department of Pharmacology, Duke University Medical Center, Durham, NC 27710.

Received for publication 15 February 1994 and in revised form 22 March 1994.

J. Clin. Invest.

(c) The American Society for Clinical Investigation, Inc.

0021-9738/94/08/0696/07 $\$ 2.00$

Volume 94, August 1994, 696-702 choline has rendered it virtually impossible to evaluate directly how the disease affects levels or turnover of this transmitter. Accordingly, studies have focused on choline acetyltransferase (acetyl-CoA:choline $O$-acetyltransferase, EC 2.3.1.6), the enzyme that synthesizes acetylcholine from choline and acetyl CoA, and on the high affinity choline transporter that takes up choline from the synapse and translocates it to the presynaptic terminal. Although nearly all investigations show that Alzheimer's disease is characterized by loss of choline acetyltransferase (1-3), this enzyme is not the rate-limiting step in acetylcholine biosynthesis and its activity is not affected by changes in neuronal activity (4-6); thus, the reduction of choline acetyltransferase activity substantiates the loss of cholinergic nerve terminals but gives no indication of the state of cholinergic function of the remaining terminals.

The issue of cholinergic function in brain regions damaged by Alzheimer's disease is vital to understanding both the origins and potential therapy of cognitive impairment. The loss of dopaminergic function in Parkinson's disease does not lead to overt symptoms until the majority of nerve terminals has degenerated and even then can be offset by administration of the dopamine precursor, L-dopa (7); the treatment enables sufficient dopamine synthesis to keep pace with increased impulse activity that offsets the deficiency in the number of nerve terminals. In contrast, administering choline, the precursor to acetylcholine, has produced variable but largely disappointing results in Alzheimer's disease (for review see reference 3), despite the fact that deficits in choline acetyltransferase indicate a smaller synaptic loss than the dopaminergic deficit in Parkinson's disease (8). This disparity suggests that the cholinergic neurons have a limitation in their ability to acquire or use choline (1) that transcends the straightforward strategy of simply providing an increase in dietary intake of the appropriate neurotransmitter precursor. To an extent, this reflects basic differences in the biosynthetic steps leading to formation of dopamine as compared with acetylcholine. Dopamine is formed from L-dopa by L-aromatic amino acid decarboxylase, which is in plentiful supply in a wide variety of cells. Acetylcholine can be formed from choline only in cholinergic neurons. Therefore, it is of critical importance that the rate-limiting step in acetylcholine synthesis is the uptake of choline from the synapse and that the activity of the high affinity transporter, located solely on cholinergic nerve terminals, is directly affected by neuronal impulses (4-6). In animal studies, upregulation of choline transport is readily obtained after manipulations that enhance cholinergic tone $(4,6,9,10)$. However, this step has rarely been evaluated in Alzheimer's disease because measurement of choline uptake requires the isolation of nerve terminal particles (synaptosomes) that maintain their viability for only a short period after death; indeed, the component of choline transport specifically associated with increased neuronal impulse activity decays the most rapidly (11). By ob- 
taining synaptosomes within $2 \mathrm{~h}$ postmortem, we have recently found that choline uptake in cortical regions of patients with Alzheimer's disease is not decreased, but rather is elevated (12); in keeping with the rapid decay of the impulse-activity-related component of uptake, the elevation is not found when the postmortem wait is prolonged $(2,13)$. These results suggest that, in Alzheimer's disease, the remaining cholinergic nerve terminals are attempting to compensate for synaptic loss by increasing their capacity to take up choline, yet, unlike the situation for dopaminergic function in Parkinson's disease, they fail to make up for the deficit.

A number of uncertainties exist in evaluating the origin and meaning of the changes in high affinity choline uptake in Alzheimer's disease. The uptake process involves not only the activity of the choline transporter itself, but also the metabolic status of the terminal (to provide the driving energy for transport), factors influencing the choline concentration gradient (removal of free cytoplasmic choline by its incorporation into acetylcholine and phospholipids), packaging of acetylcholine in synaptic vesicles, and leakage of choline back into the synapse. As a first step in delineating the contribution of each of these factors, the current study evaluates expression of the transporter, as evaluated with the specific radioligand, $\left[{ }^{3} \mathrm{H}\right]$ hemicholinium- 3 (14), in tissues obtained after rapid autopsy. We have contrasted $\left[{ }^{3} \mathrm{H}\right]$ hemicholinium-3 binding with effects on choline acetyltransferase activity in cortical regions affected by Alzheimer's disease and in a region (putamen) that does not show the characteristic cholinergic degeneration.

\section{Methods}

Patient selection and diagnostic criteria. 40 patients from the Rapid Autopsy Program, a component of the Duke University Joseph and Kathleen Bryan Alzheimer's Disease Research Center, were included in this study: 24 with Alzheimer's disease (two with coexisting Pick's disease) and 16 controls (1 with amyotrophic lateral sclerosis, 1 with gliosis, and 5 with cancers not involving the nervous system). None of the patients received anticholinergic or antihistaminic medications during the week before death. The methods for patient recruitment into the program and the standard protocols for terminal care (including 100\% oxygen by nasal prongs and oral or intravenous fluids) were approved by the Duke University Medical Center Institutional Review Board.

To meet the criteria for rapid autopsy, the postmortem delay from the time of death to freezing of tissues on dry ice was under $4 \mathrm{~h}$; in fact, only two samples were obtained at more than $2 \mathrm{~h}$ postmortem. Approximately $1 \mathrm{~g}$ of gray matter was removed from the superior frontal gyrus, the superior parietal lobule, and the putamen. Adjacent sections from each neocortical area were submitted for histological analysis by techniques described previously (12); paraffin sections were stained with hematoxylin and eosin with a Luxol fast blue counterstain for myelin, a silver stain for neuritic plaques and neurofibrillary tangles, and a Congo red stain for amyloid. By these criteria, the Alzheimer's disease group could be clearly distinguished from the controls (cortical plaques/ $\mathrm{mm}^{2}=44 \pm 3$ vs. $1 \pm 2$ in controls, $P<0.0001$; cortical tangles $/ \mathrm{mm}^{2}$ $=9 \pm 2$ vs. $0 \pm 0$ in controls, $P<0.0005$ ).

Tissue preparation and biochemical assays. Tissues were stored at $-80^{\circ} \mathrm{C}$ until analysis; preliminary studies showed no degradation of choline acetyltransferase activity or $\left[{ }^{3} \mathrm{H}\right]$ hemicholinium- 3 binding characteristics after storage (data not shown); in addition, samples were compared from the same patients in a previous study of choline acetyltransferase activity (12), and activity was found to be unchanged after as much as $7 \mathrm{yr}$ of storage. Whenever possible, samples from patients in the control and Alzheimer's disease groups were run concurrently.

Tissues were thawed in $19 \mathrm{vol}$ of ice-cold $10 \mathrm{mM}$ sodium-potassium phosphate buffer ( $\mathrm{pH}$ 7.4) and homogenized with a Polytron (Brinkmann
Instruments, Inc., Westbury, NY). Depending upon region, the tissue homogenate was diluted as much as eightfold before performing choline acetyltransferase assays, in order to ensure that enzyme activity measurements would remain linear over the incubation time used. Assays were conducted essentially as described by Lau et al. (15), using 30 $\mu \mathrm{l}$ of diluted homogenate in a total volume of $60 \mu \mathrm{l}$ containing final concentrations of $60 \mathrm{mM}$ sodium phosphate (pH 7.9), $200 \mathrm{mM} \mathrm{NaCl}$, $20 \mathrm{mM}$ choline chloride, $17 \mathrm{mM} \mathrm{MgCl}, 1 \mathrm{mM}$ EDTA, $0.2 \%$ Triton X100, $0.12 \mathrm{mM}$ physostigmine (Sigma Chemical Co., St. Louis, MO), $0.6 \mathrm{mg} / \mathrm{ml}$ bovine serum albumin (Sigma Chemical Co.), and $50 \mu \mathrm{M}$ $\left[{ }^{14} \mathrm{C}\right]$ acetyl-coenzyme A (DuPont Medical Products, Wilmington, DE; $\mathrm{sp}$ act $56 \mathrm{mCi} / \mathrm{mmol}$, diluted with unlabeled compound to $6.3 \mathrm{mCi} /$ $\mathrm{mmol}$ ). Blanks contained water instead of the tissue homogenate. Samples were preincubated for $15 \mathrm{~min}$ on ice and transferred to a $37^{\circ} \mathrm{C}$ water bath for $30 \mathrm{~min}$, and the reaction was terminated by placing the samples on ice; the labeled acetylcholine was then extracted and counted. The original homogenate was also analyzed for total protein (16), and the enzyme activity was expressed as micromoles of acetylcholine formed per milligram of protein per hour. The daily consistency and linearity of the assay was verified by including samples prepared from rat cerebral cortex.

To assess $\left[{ }^{3} \mathrm{H}\right]$ hemicholinium- 3 binding (DuPont Medical Products; sp act $121 \mathrm{Ci} / \mathrm{mmol})$, the tissue homogenate was diluted fourfold and sedimented at $40,000 \mathrm{~g}$ for $15 \mathrm{~min}$, and the supernatant solution was discarded. The membrane pellet was resuspended (Polytron) in 5-10 parts of buffer relative to the original weight of tissue and resedimented, and the resultant pellet was resuspended using a smooth glass homogenizer fitted with a Teflon pestle, in $10 \mathrm{mM}$ sodium-potassium phosphate (pH 7.4) plus $150 \mathrm{mM} \mathrm{NaCl}$, to provide a final protein concentration of $\sim 5-10 \mathrm{mg} / \mathrm{ml}$. $\left[{ }^{3} \mathrm{H}\right] \mathrm{Hemicholinium}-3$ binding was then determined essentially as described by Vickroy et al. (14), using tissue membrane preparation containing $\sim 1-2 \mathrm{mg}$ of protein in a final volume of $1 \mathrm{ml}$ of sodium-potassium phosphate $/ \mathrm{NaCl}$ buffer and ligand concentrations ranging from 0.25 to $8 \mathrm{nM}$. Incubations lasted $20 \mathrm{~min}$ at $25^{\circ} \mathrm{C}$ and were stopped by dilution with $5 \mathrm{ml}$ of ice-cold buffer, followed by rapid vacuum filtration onto Whatman GF/C filters (Whatman Inc., Clifton, $\mathrm{NJ}$ ) (presoaked for $30 \mathrm{~min}$ with $0.1 \%$ polyethyleneimine in buffer), which were then washed twice with additional buffer. The nonspecific component was defined as radioligand binding in the presence of an excess concentration $(10 \mu \mathrm{M})$ of unlabeled hemicholinium-3 (Sigma Chemical Co.) and was typically $10 \%$ of the total in a region enriched in cholinergic projections (putamen), but as much as $65 \%$ in regions relatively poor in cholinergic terminals (cortical regions). Binding values were calculated as femtomoles bound per milligram of membrane protein to correct for any differences in recovery of membranes from patient to patient. All samples were evaluated in duplicate or triplicate for both total and nonspecific binding and $K_{\mathrm{d}}$ and maximum binding capacity $\left(B_{\max }\right)^{\prime}$ values determined for each preparation.

In addition to measuring choline acetyltransferase activity and $\left[{ }^{3} \mathrm{H}\right]-$ hemicholinium- 3 binding capacity, the ratio of these two measures was also calculated to provide an index of the change in choline transporter sites (binding) relative to the loss of cholinergic nerve terminals (enzyme activity) $(12,17-20)$.

Data analysis. Data are presented as means \pm standard errors of the mean. All variables were first subjected to a three-factor ANOVA (factors of diagnostic category, region, and sex; data were log-transformed whenever variance was heterogeneous). Because none of the variables indicated a significant interaction of sex $\times$ diagnosis, or of sex $\times$ diagnosis $\times$ region, data from males and females were combined, and a twofactor ANOVA (diagnosis, region) was run. Where this test indicated a significant effect of diagnosis or a significant interaction of diagnosis $\times$ region, a subsequent lower-order analysis (Fisher's protected least significant difference) was conducted to evaluate differences attributable to diagnosis within each region. In addition, effects in the two cortical regions were compared to each other (two-factor ANOVA) to determine

1. Abbreviation used in this paper: $B_{\max }$, maximum binding capacity. 
Table I. Characteristics of the Patient Population

\begin{tabular}{lccc}
\hline \multicolumn{1}{c}{ Variable } & Control & $\begin{array}{c}\text { Alzheimer's } \\
\text { disease }\end{array}$ & Significance \\
\hline $\begin{array}{l}\text { Males/Females } \\
\text { Age }\end{array}$ & $11 / 5$ & $8 / 16$ & $P<0.03$ \\
$\quad$ Mean \pm SE & & & \\
$\quad$ Range & $67 \pm 2$ & $79 \pm 2$ & $P<0.0002$ \\
$\begin{array}{l}\text { Postmortem delay (min) } \\
\text { Mean } \pm \text { SE }\end{array}$ & $48-79$ & $57-93$ & \\
Range & $89 \pm 13$ & $57 \pm 2$ & $P<0.007$ \\
& $49-258$ & $36-83$ & \\
& & & \\
\end{tabular}

whether diagnostic effects could be distinguished in frontal versus parietal cortex, and each cortical region was compared separately with the putamen in a similar fashion. The number of males and females in each group was compared using Fisher's exact test. For all tests, significance was assumed at $P<0.05$.

Because of differences in sex ratio, postmortem delay time, and age between the Alzheimer's disease and control groups (see below), multivariate analysis was also conducted to determine whether differences in these variables significantly affected either choline acetyltransferase activity or $\left[{ }^{3} \mathrm{H}\right]$ hemicholinium-3 binding activity. In addition, regression was used to obtain values for the control group extrapolated to the sex ratio, average postmortem delay time, and average age of the Alzheimer's disease group. In no case did these corrections offset any of the significant differences between the two diagnostic categories.

Scatchard plots of $\left[{ }^{3} \mathrm{H}\right]$ hemicholinium- 3 binding were fitted by linear regression analysis, and the $K_{\mathrm{d}}$ and $B_{\max }$ values were calculated from the slope and abscissa intercept, respectively.

\section{Results}

The limited availability of preselected individuals and the rigorous requirements to enable rapid autopsy to take place do not provide a prospective framework for specific matching of patient variables. Accordingly, the population of controls available in this study differed in several aspects from the population with a diagnosis of Alzheimer's disease (Table I). First, whereas the Alzheimer's disease cohort had a predominance of females, the controls had more males. In addition, the controls tended to be slightly younger, and tissues were obtained with a somewhat longer postmortem delay time. Accordingly, where biochemical variables exhibited significant differences between the two cohorts, regression analyses also were conducted to evaluate whether these three potential confounding factors contributed to the differences (see below).

In keeping with the major cholinergic defect associated with Alzheimer's disease (12, 21-23), choline acetyltransferase activity was severely reduced in cortical regions, whereas the putamen, a region generally unaffected by the disease, displayed normal values for enzyme activity (Fig. 1). Over the age range covered by these patients, enzyme activity was not significantly correlated with age $(r=0.04$, NS); similarly, neither sex $(r$ $=0.06, \mathrm{NS})$ nor postmortem delay time $(r=0.03, \mathrm{NS})$ contributed to the difference in values. To ensure that summing up of the small differences attributed to these variables did not account for the difference in choline acetyltransferase activity between control and Alzheimer's disease groups, we used multiple regression to calculate a value for control enzyme activity matched to the sex ratio, age, and postmortem delay of the Alzheimer's disease group. This procedure did not change the
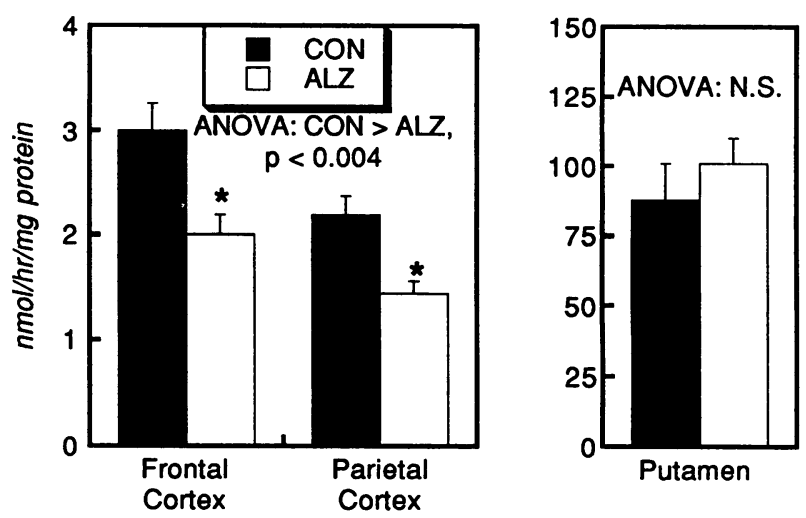

Figure 1. Choline acetyltransferase activity in rapid autopsy tissue obtained from controls (CON) and patients with Alzheimer's disease $(A L Z)$. Two-factor ANOVA across all three regions indicates a significant main effect of diagnosis $(P<0.009)$ and interaction of diagnosis $\times$ region $(P<0.005)$. ANOVAs conducted separately for the two cortical regions and for the putamen are shown within the panels, and asterisks denote significant differences from corresponding control values. In addition, the effect in the frontal cortex is distinguishable from that in the putamen (interaction of diagnosis $\times$ region, $P<0.04$ ), as is the effect in the parietal cortex $(P<0.03)$. Effects in frontal cortex are equivalent to those in parietal cortex (no interaction of diagnosis $\times$ region).

conclusions; calculated values for frontal cortex, parietal cortex, and putamen were $2.9,1.9$, and $84 \mathrm{nmol} / \mathrm{h}$ per $\mathrm{mg}$ protein, respectively, which were not significantly different from the actual control values but were significantly different from the cortical values in the Alzheimer's group (frontal cortex, $P$ $<0.001$; parietal cortex, $P<0.005$; putamen, NS).

Although nonspecific binding was as much as $65 \%$ in membrane preparations from cortical regions, reliable Scatchard determinations of the binding of $\left[{ }^{3} \mathrm{H}\right]$ hemicholinium- 3 to the high affinity choline transporter were obtained nonetheless (Fig. 2), in large part because of the plentiful amount of tissue available from human brain. Despite the loss of cortical cholinergic nerve terminals exemplified by the decline in choline acetyltransferase activity, the number of binding sites $\left(B_{\max }\right)$ was enhanced significantly in the frontal cortex of the Alzheimer's disease group (Fig. 3). There was no change in the putamen, a region presumptively not involved in the cholinergic degeneration associated with Alzheimer's disease; values in the parietal cortex were not distinguishable either from those in frontal cortex or putamen. As was true for choline acetyltransferase activity, these conclusions were unchanged when multiple regression was used to identify whether the intergroup differences in sex $(r=0.07$, NS), age ( $r=0.05, \mathrm{NS}$ ), and postmortem delay ( $r=0.02, \mathrm{NS})$ contributed to the apparent effect of the disease. Extrapolating control values matched to the sex, age, and postmortem delay of the Alzheimer's disease group produced calculated values for $B_{\max }$ of $16 \mathrm{fmol} / \mathrm{mg}$ protein in frontal cortex, 16 in parietal cortex, and 268 in putamen; using these values to determine significant differences led to the same conclusion, namely a significant increase in $B_{\max }$ caused by the disease in frontal cortex $(P<0.02)$ but not in the other regions.

The binding affinity $\left(K_{d}\right)$ of $\left[{ }^{3} \mathrm{H}\right]$ hemicholinium-3 to the choline transporter was not significantly altered by Alzheimer's disease (Fig. 4), and again sex $(r=-0.08$, NS), age $(r=0.03$, 

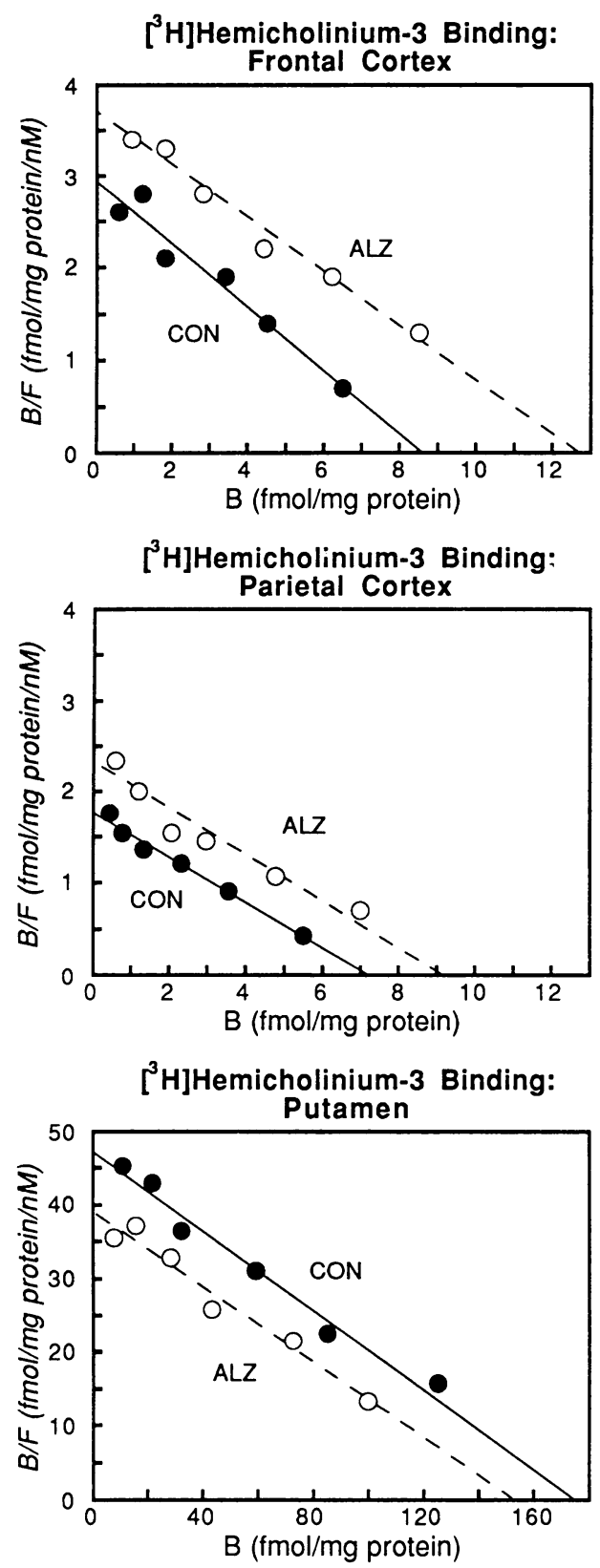

Figure 2. Representative Scatchard plots of $\left[{ }^{3} \mathrm{H}\right]$ hemicholinium-3 binding isotherms from controls ( $C O N)$ and patients with Alzheimer's disease $(A L Z)$ for each region.

NS), and postmortem delay ( $r=0.04$, NS) did not contribute to any apparent differences in the two populations.

Measurement of the binding capacity of $\left[{ }^{3} \mathrm{H}\right]$ hemicholinium3 in each region does not by itself give a complete picture of the degree of upregulation of transporter expression. because the values are manifested against a background of loss of nerve terminals in cortical regions, but not in the putamen. Accordingly, we also calculated the ratio of the $B_{\max }$ of $\left[{ }^{3} \mathrm{H}\right]-$ hemicholinium-3 binding to choline acetyltransferase activity. a relative measure of transporter expression per nerve terminal; this procedure is analogous to that previously validated for assessing choline transport per nerve terminal $(12,18)$ and for development of transporter binding sites in developing rat brain
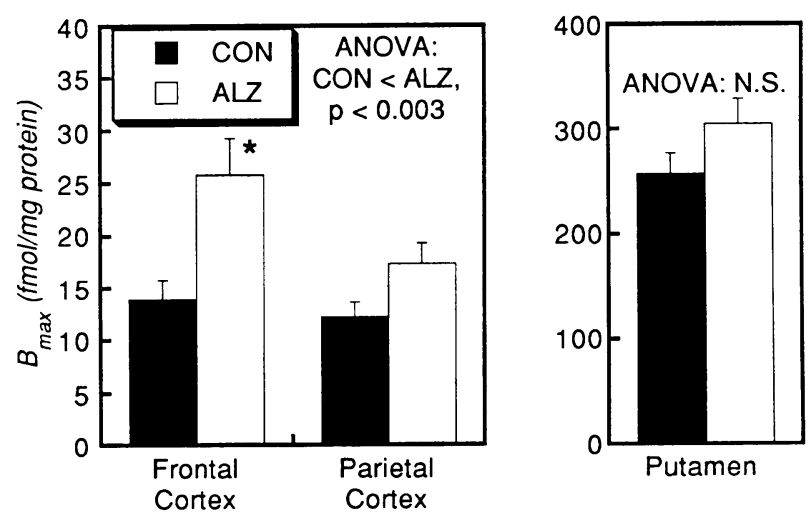

Figure 3. $\left[{ }^{3} \mathrm{H}\right]$ Hemicholinium-3 binding capacity $\left(B_{\max }\right)$ in rapid autopsy tissue obtained from controls (CON) and patients with Alzheimer's disease $(A L Z)$. Two-factor ANOVA across all three regions indicates a significant main effect of diagnosis $(P<0.003)$. ANOVAs conducted separately for the two cortical regions and for the putamen are shown within the panels, and the asterisk denotes a significant difference from the corresponding control value. In addition, the effect in the frontal cortex is distinguishable from that in the putamen (interaction of diagnosis $\times$ region, $P<0.05$ ), but the effect in the parietal cortex is not distinguishable from either that in the putamen or in the frontal cortex.

(19. 20). Expressed as a ratio, the robust upregulation of transporter sites was clearly present in both cortical regions but not in the putamen (Fig. 5). The effect in parietal cortex was not distinguishable from that in frontal cortex, but both cortical regions were collectively and individually distinguishable from the lack of upregulation in the putamen. Multiple regression analysis showed that the sex, age, and postmortem delay differences between the control and Alzheimer's disease populations did not account for the differences $(r=-0.005,0.02$, and -0.02 , respectively; all NS), nor did the summation of small contributions of these variables invalidate the conclusions. Control values extrapolated from the sex, age, and postmortem delay values found in the Alzheimer's disease group were 4.4 in fronial cortex. 7.2 in parietal cortex, and 2.8 in putamen: the cortical regions thus remained significantly different between the two

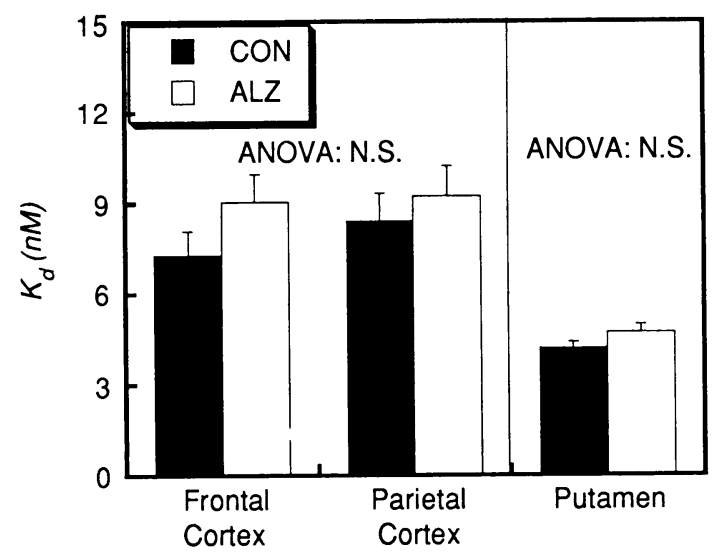

Figure $4 .\left[{ }^{3} \mathrm{H}\right] \mathrm{Hemicholinium}-3$ binding affinity $\left(K_{\mathrm{d}}\right)$ in rapid autopsy tissue obtained from controls ( $C O N)$ and patients with Alzheimer's disease ( $A L Z$ ). Two-factor ANOVA across all three regions indicates no significant differences attributable to diagnosis and no interaction of diagnosis $\times$ region. 


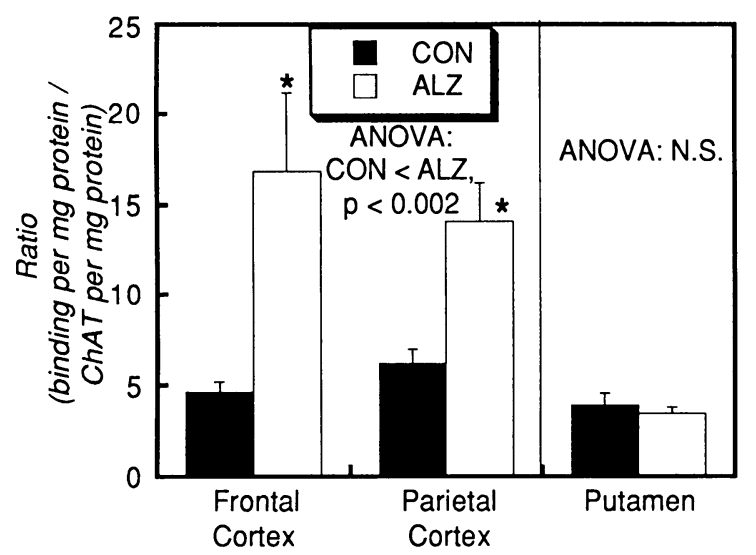

Figure 5. Ratio of $\left[{ }^{3} \mathrm{H} \mid\right.$ hemicholinium-3 binding capacity to choline acetyltransferase activity in rapid autopsy tissue obtained from controls $(C O N)$ and patients with Alzheimer's disease ( $A L Z$ ). Two-factor ANOVA across all three regions indicates a significant main effect of diagnosis $(P<0.0001)$ and an interaction of diagnosis $\times$ region $(P$ $<0.0008)$. ANOVAs conducted separately for the two cortical regions and for the putamen are shown within the panels, and asterisks denote significant differences from corresponding control values. In addition, the effect in the frontal cortex is distinguishable from that in the putamen (interaction of diagnosis $\times$ region, $P<0.0003)$, as is the effect in the parietal cortex $(P<0.004)$. Effects in frontal cortex are equivalent to those in parietal cortex (no interaction of diagnosis $\times$ region).

diagnostic categories $(P<0.01$ for both $)$ whereas the putamen was not different.

To ensure that changes in choline acetyltransferase activity or $\left[{ }^{3} \mathrm{H}\right]$ hemicholinium- 3 binding attributable to Alzheimer's disease did not reflect differences in tissue integrity or in recovery of membranes used in the binding studies, comparisons were made of total tissue protein and membrane protein (Fig. 6). Although the Alzheimer's disease group showed a significant overall elevation in total protein, the effect was quite small $(\sim 7 \%)$ and showed none of the regional selectivity characteristic of the specific cholinergic markers. Although population differences in sex, age, and postmortem delay did not produce significant correlations by themselves $(r=-0.08,0.02$, and -0.19 , respectively; all NS), the summation of these small effects actually accounted for the entire difference in total protein. Extrapolated values of the control group calculated for the age, sex, and postmortem delay in the Alzheimer's disease population produced total protein values of $106 \mathrm{mg} / \mathrm{g}$ tissue in frontal cortex, 111 in parietal cortex, and 111 in putamen, and these values were not significantly different from the Alzheimer's group. Recovery of membrane protein used in the binding studies showed no significant effect of disease.

Finally, because many of the frozen tissue samples used in this study were from the same rapid autopsy patients in whom we had previously measured high affinity choline uptake in synaptosomes from fresh tissue at the time of death (12), we were able to compare the relationship between the expression of the choline transporter $\left(B_{\max }\right.$ for $\left[{ }^{3} \mathrm{H}\right]$ hemicholinium-3) and its function (high affinity $\left[{ }^{3} \mathrm{H}\right]$ choline uptake). The correlation coefficient between the two variables was 0.69 and was highly significant $(P<0.0001)$.

\section{Discussion}

If the decline in cholinergic function in Alzheimer's disease represents simply the loss of nerve projections in the affected
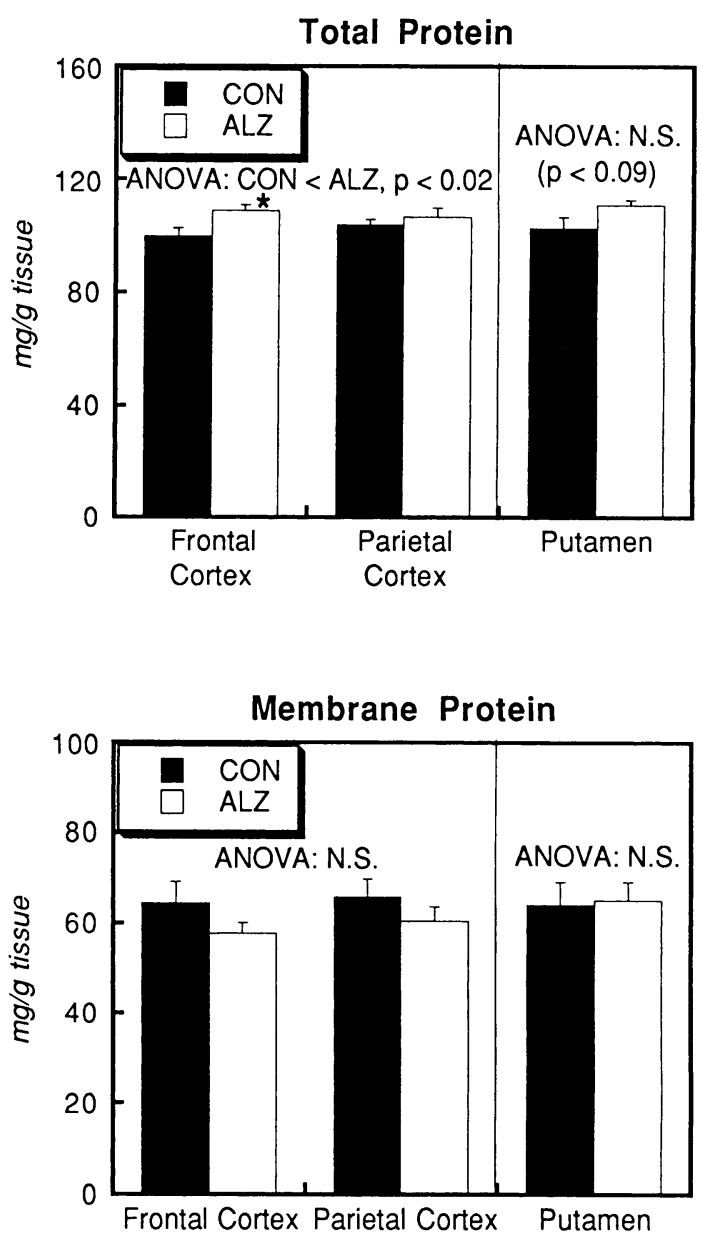

Figure 6. Total tissue protein (top) and membrane protein (bottom) in rapid autopsy tissue obtained from controls $(C O N)$ and patients with Alzheimer"s disease $(A L Z)$. For total protein, two-factor ANOVA across all three regions indicates a significant main effect of diagnosis ( $P$ $<0 .(0) 3)$. ANOVAs conducted separately for the two cortical regions and for the putamen are shown within the panels, and the asterisk denotes a significant difference from the corresponding control value. However, none of the regions show effects that are distinguishable from each other (no interactions of diagnosis $x$ region overall. or for any pair of regions). Differences for membrane protein are not significant across the three regions.

regions, without alterations in the activity or competence of the remaining terminals, then it would be expected that all cholinergic presynaptic markers would fall in parallel. Our results show definitively that this is not the case; choline acetyltransferase activity declines, but the high affinity choline transporter, as characterized by $\left[{ }^{3} \mathrm{H}\right]$ hemicholinium- 3 binding. does not, and as a result the ratio of binding to enzyme activity rises markedly in the affected regions. The absence of any change in $\left[{ }^{3} \mathrm{H}\right]-$ hemicholinium- 3 binding in the putamen, a region unaffected by the disease, indicates that there is no widespread abnormality of transporter sites as would be expected from an inherent genetic defect, but rather that the rise in binding is specifically associated with regions undergoing cholinergic degeneration. Similarly, because the putamen experiences the same potential confounding individual patient variables as the other regions. namely disparities in proximate cause of death, premortem medication history. length of the agonal state. and presence of hyp- 
oxia, the fact that both $\left[{ }^{3} \mathrm{H}\right]$ hemicholinium- 3 binding characteristics and choline acetyltransferase activity were unchanged in the putamen by Alzheimer's disease indicates that these variables do not account for the differences. The relative increase in binding in cortical regions most likely represents increased expression of the choline transporter, rather than the appearance of a different disease-linked protein capable of binding the ligand; there was no change in binding affinity as would be expected of a second binding site, and instead only the total number of sites was affected. In addition, previous work using cholinergic synaptosomes prepared from the fresh tissue at the time of death has also shown an increase in high affinity choline uptake capacity in the same regions (12). Because many of the tissues used in the current study corresponded to those for which prior work on synaptosomal uptake was conducted, we were able to correlate the changes in binding and uptake, showing that about half of the change in synaptosomal choline uptake was accountable from the change in $\left[{ }^{3} \mathrm{H}\right]$ hemicholinium- 3 binding $\left(r^{2}=0.48\right)$. This agreement is quite good when one considers that the number of transporter binding sites is a static measure that does not take into account dynamic metabolic processes that contribute to active choline uptake, such as energy gradients that provide the motive force for uptake, formation of acetylcholine and its sequestration in vesicles inside the terminal, and intraneuronal transmitter breakdown and efflux of choline. Presumably, disease-related differences in these metabolic factors account for the other half of the functional transport changes found in cortical regions from the Alzheimer's disease group as compared with controls.

The significance of the rise in $\left[{ }^{3} \mathrm{H}\right]$ hemicholinium- 3 binding relative to choline acetyltransferase would be straightforward if binding were simply preserved (i.e., unchanged) in the face of cholinergic degeneration. Because choline uptake, rather than choline acetyltransferase, is rate-limiting in acetylcholine synthesis (4-6), a decline in the number of terminals could be offset by a compensatory increase in the activity of remaining terminals and a resultant maintenance of total uptake capacity. However, we actually obtained an increase in $\left[{ }^{3} \mathrm{H}\right]$ hemicholinium- 3 binding in absolute terms, not just relative to choline acetyltransferase, suggesting that expression of transporter molecules is not just maintained in the face of neurodegeneration, but is actually increased well above the level of expression seen in normal brain. Again, this change in a static measure corresponds to a functional increase in choline transport, as indicated by direct measurement of $\left[{ }^{3} \mathrm{H}\right]$ choline uptake in synaptosomes (12). A direct proof of choline transporter overexpression in regions undergoing degeneration associated with Alzheimer's disease will have to await the cloning of the gene coding for the transporter and development of the corresponding cDNA probe.

In any case, the marked overexpression of transporter protein as evidenced by $\left[{ }^{3} \mathrm{H}\right]$ hemicholinium- 3 binding suggests that choline utilization by remaining neurons in the degenerating area is far higher than would be expected merely from compensatory increases in cholinergic neuronal activity. Indeed, animal studies confirm that cholinergic lesions alone do not produce a net upregulation of choline transporter activity in the surviving neurons (24); thus, it is unlikely that the marked increase in $\left[{ }^{3} \mathrm{H}\right]$ hemicholinium-3 binding in cortical regions in Alzheimer's disease is secondary to neurodegeneration, but rather that the change in transporter expression is a primary component of the disease. In all cells, choline is used for membrane phospholipid formation, but uniquely in cholinergic neurons, it is also used in neurotransmitter synthesis; the presence of a specific choline transporter can thus be regarded as essential to the increased demand for choline in these neurons. Wurtman (1) has proposed that a defect in the ability to use choline, in the presence of increased demand for neurotransmitter synthesis and for turnover of membrane phospholipids during membrane loss/resynthesis, leads to neuronal "autocannibalization" and sequentially to neurodegeneration. Our results are completely concordant with this view, namely that a basic defect in choline metabolism is an underlying contributor to neuron loss in Alzheimer's disease. In this scenario, inadequate incorporation of choline into membrane components and/or neurotransmitter leads to a positive feedback loop in which depletion of transmitter stores contributes to failure of neurotransmission, producing compensatory increases in impulse activity and a further increase in demand for choline. Upregulation of choline transport, while maximizing choline availability, would be effective only if sufficient choline is available and would not correct the underlying defect in intracellular choline utilization. Indeed, both free choline and phosphatidylcholine, the major choline-containing phospholipid, are deficient in Alzheimer's disease brains (25), suggesting that enhanced choline transport capabilities cannot compensate fully for the underlying defects. A further test of this relationship exists outside the framework of a neurodegenerative disease. During brain development, where increased demand for choline is associated with the need for expansion of the membrane surface in association with axonal outgrowth, the high affinity choline transporter is also overexpressed both relative to choline acetyltransferase activity and in terms of absolute activity $(19,20)$. Transporter expression during developmental membrane expansion is likewise superimposed on changes related to cholinergic nerve impulse activity $(18,20$, 26). However, unlike the case in Alzheimer's disease, the developmental overexpression is accompanied by increased choline availability (27).

Our results represent an apparent contradistinction from previous reports of $\left[{ }^{3} \mathrm{H}\right]$ hemicholinium-3 binding site in standard autopsy material, which show a general decrease in binding in cortical regions and hippocampus in Alzheimer's disease, albeit not as much a proportional decrease as is typically found for choline acetyltransferase (28). These differences are most likely attributable to short versus prolonged postmortem delay in obtaining the tissues. [ $\left.{ }^{3} \mathrm{H}\right] \mathrm{Hemicholinium-3}$ binding falls off by $20-40 \%$ in the first $4 \mathrm{~h}$ postmortem and declines significantly even with immediate cooling of the brain (29). It is therefore critical to note that, based on studies of high affinity choline uptake by the transporter, it is precisely the upregulation associated with elevated impulse activity that decays the most rapidly after death (11). Using tissue from rapid autopsy, we have shown that choline uptake is elevated in cortical regions of Alzheimer's disease patients (12), whereas after a lengthy postmortem wait, the elevation is lost $(2,13)$. The results obtained from rapid autopsy are thus more likely to correspond to the actual conditions present in the intact presynaptic terminal because they exhibit activity-related changes whereas the samples obtained after a delay do not. In concert with this interpretation, studies of $\left[{ }^{3} \mathrm{H}\right]$ hemicholinium-3 binding over a wider range of postmortem delay times $(2-29 \mathrm{~h})$ indicate a wide disparity, with some of the Alzheimer's group exhibiting normal or increased binding (28), as would be expected from samples with the shortest postmortem delays. In any case, these findings indicate the 
importance of rapid autopsy to enable appropriate interpretation of the status of cholinergic function in Alzheimer's disease. In practical terms, the finding that upregulation of $\left[{ }^{3} \mathrm{H}\right]$ hemicholinium- 3 binding parallels the functional changes seen in choline transport into intact synaptosomes (12) also means that tissues frozen after rapid autopsy can serve for future evaluations of transporter expression, structure, and function, rather than limiting inquiries to the immediate evaluation of fresh tissue. Similar effects of postmortem delay may operate on other cholinergic markers, such as choline acetyltransferase, albeit to a lesser extent than for $\left[{ }^{3} \mathrm{H}\right]$ hemicholinium-3 binding. The degree of choline acetyltransferase deficit in cortical regions of the Alzheimer's disease group seen here $(30-40 \%)$ is less than that generally found with standard autopsy material $(1-3,8)$; this suggests that the diseased tissue undergoes more rapid postmortem deterioration, in agreement with previous findings (12).

These results have significant implications for the therapy of Alzheimer's disease. If an underlying defect in choline utilization leads to failure of cholinergic neurotransmission and an ever-increasing, and possibly pathologic, upregulation of impulse activity that worsens the metabolic consequences of the defect, then the appropriate intervention is to break this positive feedback loop. Numerous clinical reports where dietary choline loading has been attempted in an effort to increase cholinergic neurotransmission have been largely disappointing (3), which would be predicted if the major defect is in choline utilization rather than choline availability. Pharmacological interventions to increase cholinergic tone in this case might be expected to produce short-term improvement in synaptic function but at the expense of accelerating neurodegeneration (1). Based on our findings, the most appropriate therapeutic strategy might be to administer agents that reduce cholinergic impulse activity and thus diminish the neuron's demand for choline; the worsening of cholinergic neurotransmission and associated cognitive impairment could instead be offset by administering drugs that augment postsynaptic cholinergic function, such as cholinergic receptor agonists and cholinesterase inhibitors. Indeed, the rigorous testing of these strategies may be the ultimate test of the cholinergic hypothesis of cognitive impairment in Alzheimer's disease or, alternatively, could establish whether the loss of noncholinergic neurons is equally important $(30,31)$.

\section{Acknowledgments}

The authors thank Dr. B. Crain, S. E. Lappi, E. C. McCook, K. Dole, and W. Smith for technical assistance.

This study was supported by U.S. Public Health Service grants MH40524 and AG-05128.

\section{References}

1. Wurtman, R. J. 1992. Choline metabolism as a basis for the selective vulnerability of cholinergic neurons. Trends Neurosci. 15:117-122.

2. Nordberg, A. 1992. Biological markers and the cholinergic hypothesis in Alzheimer's disease. Acta Neurol. Scand. 85:54-58.

3. Pepeu, G., F. Casamenti, I. M. Pepeu, and C. Scali. 1993. The brain cholinergic system in ageing mammals. J. Reprod. Fertil. 46(Suppl.): 155-162.

4. Simon, J. R., S. Atweh, and M. J. Kuhar. 1976. Sodium-dependent high affinity choline uptake: a regulatory step in the synthesis of acetylcholine. $J$. Neurochem. 26:909-922.

5. Cooper, J. R., F. E. Bloom, and R. H. Roth. 1986. The Biochemical Basis of Neuropharmacology, 5th Edition. Oxford University Press, New York. 400 pp

6. Jope, R. S. 1979. High affinity choline transport and acetylCoA production in brain and their roles in the regulation of acetylcholine synthesis. Brain Res. Rev. 1:314-344.

7. Birkmayer, W., and P. Riederer. 1983. Parkinson's Disease: Biochemistry, Clinical Pathology and Treatment. Springer-Verlag, New York. 194 pp.

8. Reisberg, B. 1983. Alzheimer's Disease: The Standard Reference. Free Press, New York. 475 pp.

9. Murrin, L. C. 1980. High-affinity transport of choline in neuronal tissue. Pharmacology (Basel). 21:132-140.

10. Cheney, D. L., J. Lehmann, C. Cosi, and P. L. Wood. 1989. Determination of acetylcholine dynamics. Drugs As Tools in Neurotransmitter Research. 12:443-495.

11. Klemm, N., and M. J. Kuhar. 1979. Post-mortem changes in high affinity choline uptake. J. Neurochem. 32:1487-1494.

12. Slotkin, T. A., F. J. Seidler, B. J. Crain, J. M. Bell, G. Bissette, and C. B. Nemeroff. 1990. Regulatory changes in presynaptic cholinergic function assessed in rapid autopsy material from patients with Alzheimer disease: implications for etiology and therapy. Proc. Natl. Acad. Sci. USA. 87:2452-2455.

13. Rylett, R. J., M. J. Ball, and E. H. Colhoun. 1983. Evidence for high affinity choline transport in synaptosomes prepared from hippocampus and neocortex of patients with Alzheimer's disease. Brain Res. 289:169-175.

14. Vickroy, T., W. Roeske, and H. Yamamura. 1984. Sodium-dependent high-affinity binding of $\left[{ }^{3} \mathrm{H}\right]$ hemicholinium- 3 in the rat brain: a potentially selective marker for presynaptic cholinergic sites. Life Sci. 35:2335-2343.

15. Lau, C., F. J. Seidler, A. M. Cameron, H. A. Navarro, J. M. Bell, J. Bartolome, and T. A. Slotkin. 1988. Nutritional influences on adrenal chromaffin cell development: comparison with central neurons. Pediatr. Res. 24:583-587.

16. Lowry, O. H., N. J. Rosebrough, A. L. Farr, and R. J. Randall. 1951 Protein measurement with the Folin phenol reagent. J. Biol. Chem. 193:265-275.

17. Nemeroff, C. B., G. Bissette, T. A. Slotkin, F. J. Seidler, B. E. Miller, and H. Ghanbari. 1991. Recent advances in the neurochemical pathology of Alzheimer's disease: studies of neuropeptides, cholinergic function and Alzheimer's disease-associated protein. Ann. NY Acad. Sci. 640:193-196.

18. Navarro, H. A., F. J. Seidler, J. P. Eylers, F. E. Baker, S. S. Dobbins, S. E. Lappi, and T. A. Slotkin. 1989. Effects of prenatal nicotine exposure on development of central and peripheral cholinergic neurotransmitter systems. Evidence for cholinergic trophic influences in developing brain. J. Pharmacol. Exp. Ther. 251:894-900.

19. Zahalka, E. A., F. J. Seidler, S. E. Lappi, E. C. McCook, J. Yanai, and T. A. Slotkin. 1992. Deficits in development of central cholinergic pathways caused by fetal nicotine exposure: differential effects on choline acetyltransferase activity and $\left[{ }^{3} \mathrm{H}\right]$ hemicholinium-3 binding. Neurotoxicol. Teratol. 14:375-382.

20. Zahalka, E., F. J. Seidler, S. E. Lappi, J. Yanai, and T. A. Slotkin 1993. Differential development of cholinergic nerve terminal markers in rat brain regions: implications for nerve terminal density, impulse activity and specific gene expression. Brain Res. 601:221-229.

21. Rossor, M., J. Fahrenkrug, P. Emson, C. Mountjoy, L. Iversen, and M. Roth. 1980. Reduced cortical choline acetyltransferase activity in senile dementia of Alzheimer type is not accompanied by changes in vasoactive intestinal polypeptide. Brain Res. 201:249-253.

22. Sims, N. R., D. M. Bowen, S. J. Allen, C. C. T. Smith, D. Neary, D. J. Thomas, and A. N. Davison. 1983. Presynaptic cholinergic dysfunction in patients with dementia. J. Neurochem. 40:503-509.

23. Sanfeliu, C., A. Hunt, and A. J. Patel. 1990. Death of subcortical cholinergic neurons in certain neurodegenerative disorders may not be due to an overstimulation of $N$-methyl-D-aspartate receptors. Brain Res. 506:319-322.

24. Gomeza, J., C. Aragón, and C. Giménez. 1992. High-affinity transport of choline and amino acid neurotransmitters in synaptosomes from brain regions after lesioning the nucleus basalis magnocellularis of young and aged rats. Neurochem. Res. 17:345-350.

25. Nitsch, R. M., J. K. Blusztajn, A. G. Pittas, B. E. Slack, J. H. Growdon, and R. J. Wurtman. 1992. Evidence for a membrane defect in Alzheimer disease brain. Proc. Natl. Acad. Sci. USA. 89:1671-1675.

26. Shelton, D. L., J. V. Nadler, and C. W. Cotman. 1979. Development of high affinity choline uptake and associated acetylcholine synthesis in the rat fascia dentata. Brain Res. 163:263-275.

27. Zeisel, S. H. 1981. Dietary choline: biochemistry, physiology, and pharmacology. Annu. Rev. Nutr. 1:95-121.

28. Pascual, J., A. Fontán, J. J. Zarranz, J. Berciano, J. Flórez, and A. Pazos. 1991. High-affinity choline uptake carrier in Alzheimer's disease: implications for the cholinergic hypothesis of dementia. Brain Res. 552:170-174.

29. Kristofiková, Z., J. Klaschka, V. Nemcová, E. Majer, and E. Fales. 1993. Effect of postmortem storage on the $\left[{ }^{3} \mathrm{H}\right]$ hemicholinium- 3 binding site in the rat brain. Preliminary study for investigations of human patients with Alzheimer's disease. Arch. Gerontol. Geriatr. 16:117-128.

30. Whitehouse, P. J. 1993. Cholinergic therapy in dementia. Acta Neurol. Scand. 88:42-45.

31. Winblad, B., E. Messamore, C. O'Neill, and R. Cowburn. 1993. Biochemical pathology and treatment strategies in Alzheimer's disease: emphasis on the cholinergic system. Acta Neurol. Scand. 88:4-6. 